\title{
Near Wake of a Hypersonic Blunt Body with Mass Addition
}

\author{
Donald J. Collins, * Lester Lees, $\uparrow$ And Anatol Roshko $\ddagger$ \\ California Institute of Technology, Pasadena, Calif.
}

\begin{abstract}
An experimental investigation of the steady, laminar near-wake flowfield of a two-dimensional, adiabatic, circular cylinder with surface mass transfer has been made at a freestream Mach number of 6.0. The pressure and mass-concentration fields associated with the transfer of argon, nitrogen, or helium into the near wake were studied for mass transfer from the forward stagnation region, and from the base. For sufficiently low mass transfer rates from the base, for which a recirculating zone exists, the entire near-wake flowfield correlates with the momentum flux, not the mass flux, of the injectant, and the mass-concentration field is determined by counter-current diffusion into the reversed flow. For mass addition from the forward stagnation region, the pressure field is undisturbed and the mass-concentration field is nearly uniform in the region of reversed flow. The axial decay of argon mass concentration in the intermediate wake, downstream of the neck, is explained with the aid of an integral solution in the incompressible plane, from which the location of the virtual origin for the asymptotic far-wake solution has been derived as one result.
\end{abstract}

\section{Nomenclature}

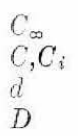

$=\left(\mu / \mu_{\infty}\right)\left(T_{\infty} / T\right)$, Chapman-Rubesin factor

$=\rho_{i} / \rho$, mass concentration

$=$ cylinder diameter

$=$ binary diffusion coefficient

$G\left(M_{\infty}\right)=\frac{P_{0}}{P_{\infty}} \frac{1}{M_{\infty}}\left[\frac{T_{\infty}}{T_{0}}\right]^{1 / 2}=\frac{1}{M_{\infty}}\left[1+\frac{\gamma-1}{2} M_{\infty}\right]^{\frac{\gamma+1}{2(\gamma-1)}}$

$H \quad=\left(\dot{m}_{i} / \dot{m}_{f}\right) G\left(M_{\infty}\right)$, Korst injection parameter

$I \quad=\left(\dot{m}_{i} / 2 \dot{m}_{B . L}.\right)\left(\mathscr{M}_{\mathrm{Air}} / \mathscr{M}_{i}\right)^{1 / 2}$ mass transfer parameter

$\dot{m}_{B . L .}=\left[\int_{0}^{\delta} \rho u d y\right]_{s}=\rho_{e} u_{e}\left(\delta-\delta^{*}\right)_{s}$, mass flux per unit span in eylinder boundary layer upstream of separation

$\dot{m}_{f}=\rho_{\infty} u_{\infty} d$, intercepted freestream mass flux per unit span

$\dot{m}_{i} \quad=$ mass flux per unit span of injectant

$\dot{H}=\dot{m}_{2} / 2 \dot{m}_{B . L .}$, mass transfer parameter

$M \quad=$ Mach number

M $=$ molecular weight

$P \quad=$ pressure

$P_{0_{2}} \quad=$ Pitot pressure, impact pressure

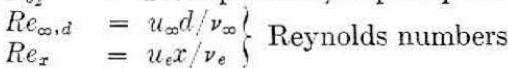

Presented as Paper 69-67 at the AIAA 7 th Aerospace Sciences Meeting, New York, January 20-22, 1969; submitted December 23, 1968; revision received July 28, 1969. The work discussed in this paper was carried out under the sponsorship and with the financial support of the U.S. Army Research Office and the Advanced Research Projects Agency, Contract DA-31-124$\mathrm{ARO}(\mathrm{D})-33$. This research is a part of Project DEFENDER sponsored by the Advanced Research Projects Agency.

* Research Fellow, Aeronautics. Member AIAA.

$\dagger$ Professor of Aeronautics. Fellow AIAA.

$\div$ Professor of Aeronautics. Associate Fellow AIAA.
$S$

$T$

$u, v$

$u^{*}$

$\tilde{U}$

$=$ temperature

$=$ physical velocity components

$=u_{\psi}=0 / u_{e}$, dividing streamline velocity ratio

$=u / u_{e}$, transformed velocity

$=\left[u(\tilde{X}) / u_{\max }\right] \cdot\left[\left|u_{\max }\right|\left(x_{r}-x_{b}\right) / D\right]=\operatorname{Re}(\tilde{X}) S c$

$=$ physical coordinates

$=\left(x-x_{b}\right) /\left(x_{r}-x_{b}\right)$, transformed physical $x$

$=\int_{0}^{x / d} \frac{\rho_{e} u_{e} \mu_{e}}{\rho_{\infty} u_{\infty} \mu_{\infty}} d\left(\frac{x}{d}\right)$, modified Howarth coordinate

$=$ location of reattachment

$=v /\left(x_{r}-x_{b}\right)$, transformed physical $y$

$=\left[C_{\infty} R e_{\infty}, d\right]^{1 / 2} \int_{0}^{y / d} \frac{\rho_{e} u_{e}}{\rho_{\infty} u_{\infty}} \frac{\rho}{\rho_{e}} d\left(\frac{y}{d}\right)$, modified Howarth coordinate

$=\left(1-4 \Delta / \tilde{U}^{2}\right)^{1 / 2}$

$=$ specific heat ratio

$=$ boundary-layer thickness

= base boundary-layer thickness

$=\int_{0}^{\delta}\left(1-\frac{\rho u}{\rho_{e} u_{e}}\right) d y$, boundary-layer displacement

$=F(0)^{\prime \prime} / \sigma(\tilde{X})^{2}$, self-similar profile curvature at $\tilde{Y}=0$

$\theta \quad=\int_{0}^{\delta} \frac{\rho u}{\rho_{e} u_{e}}\left(1-\frac{u}{u_{e}}\right) d y$, momentum thickness; angle from forward stagnation point

$\nu \quad=$ kinematic viscosity coefficient

$\xi \quad=X-X_{N}$, transformed Howarth coordinate

$\xi_{0} \quad=$ virtual origin for the asymptotic far wake

$\rho \quad=$ density

$\sigma(\tilde{X}), \sigma(X)=$ nondimensional length scale for the self-similar profiles

$\psi \quad=$ stream function 


\section{Subscripts}

$b=$ base stagnation point

屯 = centerline

$e=$ external

$i \quad=$ injected fluid

$N=$ neck

$s$ = boundary layer immediately upstream of separation

$0=$ total or stagnation condition

$\infty=$ undisturbed freestream conditions

\section{Introduction}

$\mathbf{T}$ HE roles of heat and mass transfer in the development of the wake behind a body, and the diffusion of chemical species within the near- and far-wake flows are problems of considerable technological importance. The purpose of the present investigation was to determine the effect of mass addition on the laminar wake of a circular cylinder and to examine the diffusion of the injected species within the near-wake flow. The details of this work are given in Ref. 1.

The basic flowfield structure for the circular cylinder [Fig. 1], in the absence of mass addition, has previously been described in considerable detail in Refs. 2-4, and therefore only those aspects of the flow which relate directly to the phenomena of mass addition will be included here. The problem of mass diffusion in the far wake of a uniformly porous cylinder has been discussed in Refs. 5 and 6 , and Her$\mathrm{zog}^{7}$ has investigated the effects of nitrogen addition from the base on the near-wake pressure field.

It seems obvious, a priori, that mass addition into the nearwake flow from the base will perturb the flowfield structure. However, it is not obvious what effects on the near wake will be produced by mass addition from the forward stagnation region of the body when the mass transfer rates are low enough that the bow shock structure is not affected. In the latter case, one might expect perturbations to occur in the structure of the cylinder boundary layer which, after separation, becomes the free shear layer in the near wake. For the addition of a foreign species, one might also anticipate changes in the dynamics of the reattaching streamline resulting from the presence of finite mass concentrations. The accumulation of these effects might be expected to influence the near-wake flowfield according to the theories of Korst $^{8}$ and Chapman. ${ }^{9}$

For mass addition from the base, three distinct regimes may be defined for the influence of mass addition on the nearwake pressure field. These three regimes are distinguished according to whether the mass flux, the momentum flux or the volume flux of the injected species dominates the changes in the flowfield.

For negligible velocities of the injectant, the concept of base bleed has been introduced by Korst et al., ${ }^{8}$ and by Chapman. ${ }^{10}$ Numerous experiments have been reported (cf. Herzog, ${ }^{7}$ Davis, ${ }^{11}$ Carriére, ${ }^{12}$ Ginoux ${ }^{13}$ ) in which correlations have been attempted based on the empirically derived bleed parameters arising from the Chapman-Korst ideas of mass entrainment by the free shear layers bounding the recirculating flow. The arguments contained in the Chapman-Korst theory lead to the conclusion that changes in the base pressure, and hence the near-wake structure, depend on the normalized mass flux of the injected fluid. The experiments of Carriére ${ }^{12}$ and Ginoux, ${ }^{13}$ using only air as an injectant, seem to substantiate these ideas. However, correlations between the changes in the near-wake pressure field and the molecular weight of the injected fluid have not been obtained previously.

At the opposite end of the spectrum, for large velocities of the injectant, the experiments of Lewis and Behrens ${ }^{14}$ conclusively demonstrate that when the recirculating zone no longer exists in the wake the changes in the pressure field depend on the volume flow of the injectant.

In the intermediate mass transfer regime, for which a recirculating zone still exists in the near wake, a third regime exists in which the momentum flux of the injectant is comparable with the momentum flux of the reversed flow. The present experiments were designed to study this regime and to determine the physical mechanism associated with the effect of mass addition on the base pressure and the near-wake pressure field, and the correlation of these effects with Reynolds number and molecular weight by using argon, nitrogen, and helium as injectants.

Few detailed theoretical or experimental investigations have been performed to determine the distribution in the near-wake flowfield of mass species transferred to the flow from the body. $\S$ In the present investigation, the distributions of the injected species were measured in the steady near-wake flow of a circular cylinder, and the important mechanisms that determine the distribution of passive scalars (i.e., mass and, by analogy, temperature) in the near wake are examined. In addition, to understand the behavior of the asymptotic far-wake distribution of the injected species, an intermediate wake region immediately downstream of the wake neck is examined to determine the influence of the near-wake flow on the development of the asymptotic far wake.

\section{Experimental Technique}

\section{Flow Facility}

The experiments were performed at a nominal freestream Mach number of 6.0 , and freestream Reynolds numbers $R e_{\infty, d}=0.905 \times 10^{4}$ and $2.95 \times 10^{4}$ in Leg I of the GALCIT hypersonic facility. This facility is a closed return, continuous flow tunnel with a $5 \times 5$ in. test section and a stagnation temperature of $735^{\circ} \mathrm{R}$.

\section{Models}

The wind-tunnel models used in this investigation (Fig. 1) consisted of $0.200 \mathrm{in}$. diam stainless-steel tubes which spanned the test section. These models were provided with triangular cross-flow fences downstream of the cylinder to insure a spanwise uniform static-pressure field in the base region. ${ }^{1}$

Two models were used in the experiments: a pressure model and a mass-transfer model. The pressure model used an internal O-ring to hold either base mounted Pitot probes or insert static-pressure probes, with which the axial distributions of Pitot and static pressure were measured in the reversed flow in the absence of mass addition. The mass transfer model (Fig. 1) used a machineable porous ceramic insert, bonded with epoxy into a longitudinal slot in the cylinder, to transfer the injectant from the body surface into the flowfield.

To insure the spanwise uniformity of the flowfield, the model plenum pressure and temperature were measured. The plenum pressure was found to be uniform within $0.5 \%$ and independent of the external flow over the entire operating range. The gas temperature in the plenum was within $10 \%$ of the adiabatic-wall temperature of the model. Measurements of the spanwise pressure and mass-concentration distributions in the base region confirmed that the near-wake flow with mass addition was nearly two-dimensional in the region of reversed flow. Downstream of reattachment, three-dimensional effects resulted in an over-all decrease in the mass-concentration levels.

$\S$ Somewhat limited measurements have been reported by Davis. ${ }^{11}$ 


\section{Pressure Measurement}

The impact-pressure and mass-concentration fields were measured by using a family of Pitot-sampling probes. The measurements were made with a $0-5$ psia pressure transducer manufactured by Statham Instrument Co., and a vacuum reference silicone-oil micromanometer. The data were corrected for both viscous and probe interference effects, and were adjusted to account for existing tunnel gradients.

\section{Yass Concentration Measurement}

Reis and Fenn ${ }^{15}$ have demonstrated that for the flow of a binary mixture of gases of disparate molecular weights, the presence of a sampling probe may cause barotropic species separation by virtue of the existence of a strong lateral pressure gradient in the vicinity of the probe tip. The magnitude of this separation is a function of the Mach number, Reynolds number, and the disparity between the constituent molecular weights. The important parameter in the mass-sampling problem is the ratio of the area of the sampled stream tube relative to the incident cross-section of the probe, which, for the low Reynolds numbers encountered in the present experiments, is typically of the order of 0.2 , because of viscous effects.

To verify that the mass concentrations measured by the probe were correct, measurements were performed in the wake behind the cylinder, with mass addition from the base. For these measurements, the mass concentration at a point was measured as a function of probe Reynolds number and the mass flux through the probe, for argon and helium addition. No barotropic effects were found either for an increase in probe Reynolds number by a factor of five, or for a change in probe mass flux by a factor of ten. On the basis of these measurements, it is concluded that the sample obtained is representative of the undisturbed flowfield.

The detector for the mass-analysis system was a Varian Associates Partial Pressure Gauge, a small magnetic mass spectrometer utilizing a modified Bayard-Alpert source. During operation, a continuous sample was throttled to a known, constant pressure $(25 \mu)$ maintained by a trapped mechanical pump, and a portion of the sample was expanded through a fixed capillary into the spectrometer, operating at a pressure of $2 \times 10^{-6}$ torr, compared to the spectrometer background pressure of $2 \times 10^{-8}$ torr.

The spectrometer system was calibrated using known mixtures. A single peak was scanned for each binary mixture, i.e., $m / e=40$ for argon-air and $m / e=28$ for both nitrogen-air and helium-air, and the output of the spec-

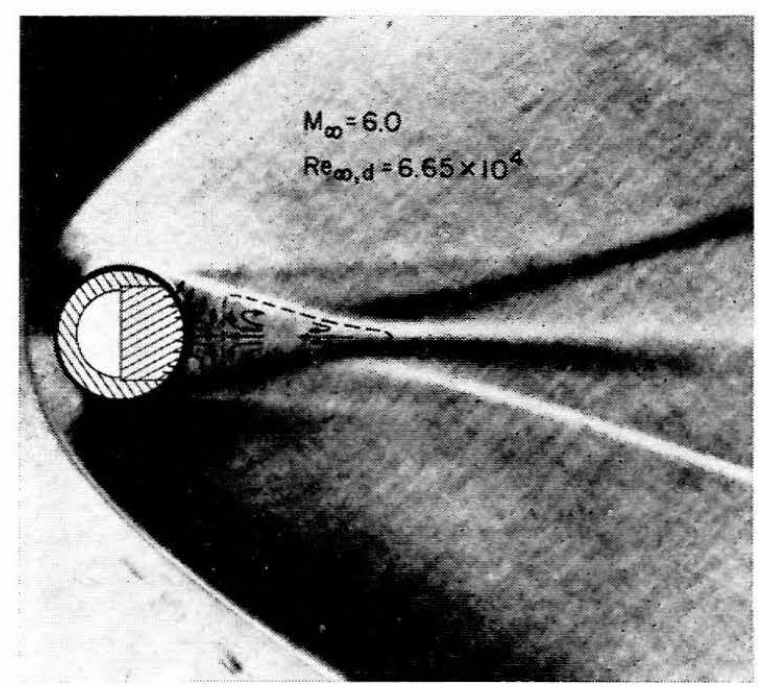

Fig. 1 The flowfield with base mass addition (Schlieren photo from Ref. 4).

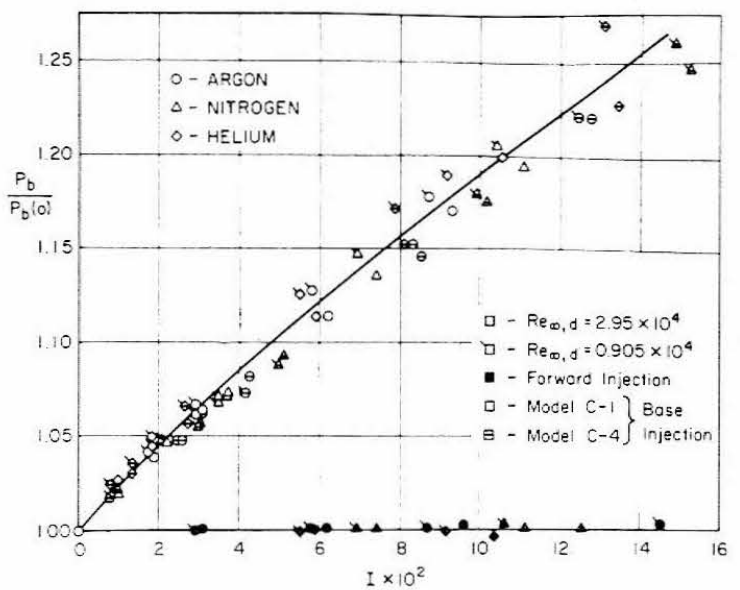

Fig. 2 Variation of the base pressure with mass addition.

trometer was recorded, normalized by the spectrometer output for the pure gas under the same inlet conditions.

For argon, the error in the measurements was at most $1.5 \%$ of the measured value over the entire range of mass concentrations. For a $1 \%$ error in the measurement of nitrogen, however, the percent error in the mass concentration of the added nitrogen becomes unbounded as zero concentration is approached, because the background gas (air) contains $\sim 80 \%$ nitrogen. The helium mass concentrations were also measured using the $m / e=28$ peak of nitrogen because of an instability which occurred in the $\mathrm{m} / e=4$ peak of helium. Therefore the error for helium also becomes unbounded for vanishing mass concentrations. However, as in the case of nitrogen, the percentage error rapidly diminishes with increasing mass concentration of helium.

\section{Structure of the Flowfield with Mass Addition}

\section{Mass Addition from the Front}

Measurements of the near-wake pressure field for mass addition from the forward stagnation region demonstrate that there is no effect on the Pitot-pressure distributions and the base pressure (Fig. 2). Neither the bow shock wave nor the Pitot-pressure distributions in the boundary layer upstream of separation are altered by mass addition over the range of conditions shown in Fig. 2. It appears that the dynamies of the flow in the near wake are unaltered. Hence the mass-concentration field is simply the field of a scalar quantity diffusing from an initial distribution in the cylinder boundary layer into the otherwise undisturbed near-wake flow. This is described in detail in a later section.

\section{Mass Addition from the Base}

\section{Base pressure}

To analyse the results of the changes in base pressure with base mass addition, the first attempt was to correlate the results with the mass flux parameter

$$
H=\left(\dot{m}_{i} / \dot{m}_{f}\right) G\left(M_{\infty}\right)
$$

proposed by Korst et al. ${ }^{8}$ The most important result of the present experiments is that such a mass flux parameter does not adequately correlate the data. The present experiments show that, under the condition that a nonvanishing recirculating flow exists in the near wake, the base pressure (Fig. 2), and the entire near-wake pressure field (Fig. 5) correlate with the injection parameter

$$
I=\left(\dot{m}_{i} / 2 \dot{m}_{B . L .}\right)\left(\Re_{\mathrm{Air} /} / \Re_{i}\right)^{1 / 2}
$$

As shown in the following section, this parameter represents the properly normalized momentum flux of the injected fluid 


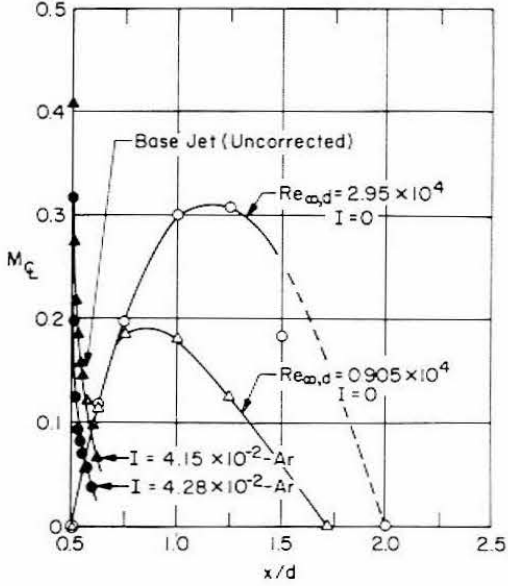

\section{Fig. 3 Axial Mach number distribution in the near wake.}

It is obvious from Fig. 2 that if the Korst parameter is used when the molecular weight differs from that of air, that no correlation would be obtained.

The present experiments suggest a physical model for the base pressure which depends on the dynamics of the interaction of the injected gas with the recirculating near-wake flowfield. This model possesses two aspects: 1) the interaction of the injected fluid with the reversed flow forms a stagnation point off of the body, and consequently 2) the injected fluid is turned away from the axis and is impressed on the free shear layer near its origin, inducing an increase in pressure. Both aspects are coupled and result in a momentum flux dependence for the base pressure.

The primary aspect of this model is the interaction between the injected fluid and the reversed flow. For moderate mass addition rates, a recirculating vortex remains in the near wake, and hence two stagnation points exist on the axial streamline: the rear stagnation point, and a stagnation point in the vicinity of the base (see Fig. 1). Figure 3 demonstrates that the total pressure of the injected gas is comparable with the total pressure on the centerline of the recirculating vortex, and hence the base stagnation point is formed off the body by a balance between the total pressures of the two interacting streams. The generalization of the base pressure, or characteristic near-wake pressure, is the stagnation pressure of the highly dissipative reversed flow at the base stagnation point, $\uparrow$ and is therefore determined by the momentum flux of the injected fluid. The proper scaling for this momentum flux is given by the momentum flux of the reversed flow.

The total pressure on the axial streamline decays monotonically from its value at the rear stagnation point to its value at the base stagnation point as a consequence of viscous dissipation. Therefore, as the momentum flux of the injected gas, and hence its total pressure, is increased, the base stagnation point moves toward the rear stagnation point into a region of increasing total pressure for the reversed flow. Simultaneously, the reversed flow is reduced as a consequence of the reduction of the value of $u^{*}$ on the stagnating streamline. For these reasons, the base pressure increases with increasing mass addition. When the total pressure of the injected fluid exceeds the maximum available total pressure in the reversed flow, the entrainment required by the shear layers is satisfied by the injected gas and the reversed flow vanishes, as reported by Herzog. ${ }^{7}$

The second aspect of the model involves the interaction between the injected gas and the separating free shear layer. The gas injected from the base is turned by the reversed flow in a distance $\delta_{b}=\delta_{b}\left(m_{i} /\left[\mathfrak{M}_{i}\right]^{1 / 2}\right)$, the distance from the

- This distinction has not been made in the work of Fuller and Reid, ${ }^{16}$ for example, and care must be used in the interpretation of their results. body to the base stagnation point, and is impressed on the separating free shear layer over a distance of the order of $\delta_{b}$. The effects on the free shear layer caused by the impressed mass are determined by the amount of mass that the layer is required to entrain. The idealization proposed by Chapman $^{17}$ gives an upper bound on the mass entrainment resulting from viscous stresses. If the entrainment is greater than Chapman's value, viscous stresses are no longer sufficient to turn the flow and a pressure increase is induced by the interaction which turns both the impressed flow and the flow in the free shear layer. The amount of the turning of the shear layer flow, and hence the induced pressure, is determined from a balance between the momentum flux of the impressed flow and some characteristic momentum flux associated with the free shear layer.

For the present experiments, the entrainment rate of the impressed fluid,

$$
-\zeta_{0}=\lambda_{e}\left(R e_{x} / C_{\infty}\right)^{1 / 2}
$$

where

$$
\lambda_{e}=\rho_{-\infty} v_{-\infty} / \rho_{e} u_{e}
$$

exceeds the Chapman value by a factor of from four to eight. Therefore, so long as the entrainment is large compared to the entrainment specified by the Chapman solution, and so long as a recirculating vortex exists in the near wake, the change in the near-wake pressure field with base mass addition will be determined by the properly normalized momentum flux of the injected gas.

\section{Mass transfer parameter}

From the concept of the interaction of the injected fluid with the reversed flow, it is clear that the change in the nearwake pressure field is determined by a balance between the momentum flux of the injectant and the momentum flux of the highly dissipative reversed flow at the base stagnation point. However, for the circular cylinder, because the subcritical separation process allows a smooth transition from the cylinder boundary laver to the nonsimilar laminar free shear layer, the Reynolds number scaling is the same for both the reversed flow and the cylinder boundary layer. Therefore, to facilitate the calculations, the characteristic near-wake momentum flux has been taken as that associated with the cylinder boundary layer up-stream of separation.

Using these ideas, the injection parameter is formulated from the ratio of the momentum flux of the injected gas to the momentum flux in the boundary layer upstream of separation, given by

$$
\pi=\frac{\dot{m}_{i} u_{i}}{2\left(\rho_{e} u_{e}^{2}\right)_{s} \int_{0}^{\delta}\left(\frac{\rho u^{2}}{\rho_{e} u_{e}^{2}}\right)_{s} d y}
$$

Evaluating the integral,

$$
\pi=\dot{m}_{i} u_{i} / 2\left(\rho_{e} u_{e}^{2}\right)_{s}\left(\delta-\delta^{*}-\theta\right)_{s}
$$

Introducing the boundary-layer mass flux,

$$
\dot{m}_{B . L .}=\left[\rho_{e} u_{\epsilon} \int_{0}^{\delta}\left(\frac{\rho u}{\rho_{\epsilon} u_{e}}\right) d y\right]_{s}=\left[\rho_{\epsilon} u_{\epsilon}\left(\delta-\delta^{*}\right)\right]_{s}
$$

and substituting the Mach numbers for the two flows, the expression becomes

$\pi=\frac{\dot{m}_{i}}{2 \dot{m}_{B . L}}\left[\frac{\Re_{\mathrm{Air}}}{\mathfrak{M}_{i}}\right]^{1 / 2}\left\{\frac{M_{i}}{M_{\epsilon}}\left[\frac{\delta-\delta^{*}}{\delta-\delta^{*}-\theta}\right]\left[\frac{\gamma_{i} T_{i}}{\gamma_{\epsilon} T_{\epsilon}}\right]^{1 / 2}\right\}_{\delta}$

To this point, no assumptions have been made about the nature of the flows.

In the present experiments it is most convenient to evaluate the momentum flux of the injectant at the base by considering the flow through the porous ceramic. The model plenum pressure was measured and found to be independent 
of the external pressure over the entire operating range. Further, the pressure ratio across the ceramic, $30 \leq P_{0 i} /$ $P_{0} \leq 360$, greatly exceeds the pressure ratio required for a choked flow through the ceramic for the entire range of the parameter I given in Fig. 2. Similar results have been reported by Shreeve, ${ }^{18}$ who found choking for $P_{0 \mathrm{i}} / P_{b} \geq 10$. In addition, measurements of the gas temperature in the model plenum, and order of magnitude estimates of the heat transfer to the gas from the ceramic imply that the flow through the porous ceramic is an isothermal, choked flow. As a consequence, $M_{i} \rightarrow \gamma_{i}^{-1 / 2} ; T_{i} \rightarrow T_{0_{3}} \approx T_{a x}$, the adiabatic recovery temperature of the model. Then, in Eq. ( 7 )

$$
\frac{M_{i}}{M_{e}}\left[\frac{\gamma_{i} T_{i}}{\gamma_{e} T_{e}}\right]^{1 / 2} \rightarrow \frac{1}{M_{c}}\left[\frac{1}{\gamma_{c}} \frac{T_{a v}}{T_{0}} \frac{T_{0}}{T_{e}}\right]^{1 / 2}
$$

independent of the injected fluid.

Therefore,

$\pi=\frac{\dot{m}_{i}}{2 \dot{m}_{B . L}}\left[\frac{\Re_{\mathrm{Air}}}{\mathfrak{M}_{i}}\right]^{1 / 2}\left\{\frac{1}{M_{e}}\left[\frac{\delta-\delta^{*}}{\delta-\delta^{*}-\theta}\right]\left[\frac{1}{\gamma_{e}} \frac{T_{0_{i}}}{T_{0}} \frac{T_{0}}{T_{e}}\right]^{1 / 2}\right\}_{s}$

The momentum flux ratio ean be rewritten as $\pi=\left\{F\left(M_{e}\right.\right.$, $\left.\left.\gamma_{e}\right)\right\} I$, where

$$
I=\left(\dot{m}_{i} / 2 \dot{m}_{B . L .}\right)\left[\Re_{\mathrm{Air} /} / 9 \pi_{i}\right]^{1 / 2}
$$

and where

$$
F\left(M_{\epsilon}, \gamma_{\epsilon}\right)=\left\{\frac{1}{M_{e}}\left[\frac{\delta-\delta^{*}}{\delta-\delta^{*}-\theta}\right]\left[\frac{1}{\gamma_{e}} \frac{T_{0 i}}{T_{0}} \frac{T_{0}}{T_{e}}\right]^{1 / 2}\right\}_{s}
$$

For the circular cylinder at large Reynolds numbers and hypersonic Mach numbers, $F\left(M_{e}, \gamma_{\epsilon}\right)$ is dependent on $R e_{\infty, d}$ only through the weak dependence of the location of the separation point on $R e_{\infty, d}$. Therefore $F\left(M_{e}, \gamma_{e}\right)$ is assumed to be invariant. The boundary-layer mass flux per unit span upstream of separation was calculated from a locally similar solution given by Klineberg. ${ }^{3}$

The present mass transfer criterion differs from those derived from the models proposed by Korst et al. ${ }^{8}$ and by

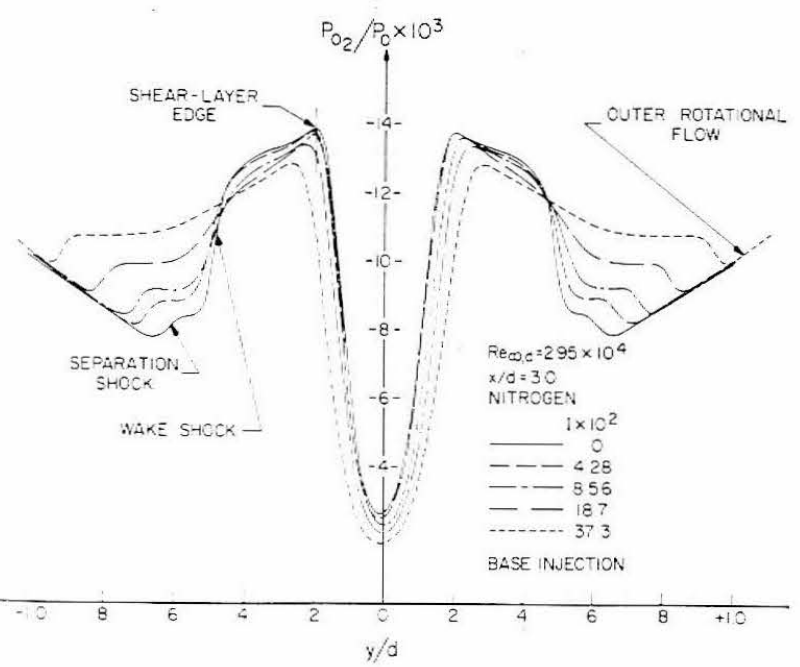

Fig. 4 The influence of increasing mass addition on the near-wake flowfield.

T Note: If the process is adiabatic, then

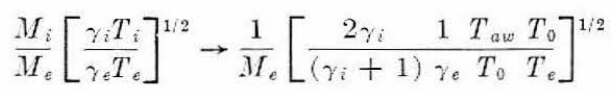

The results for adiabatic and isothermal flow differ by $8 \%$ for nitrogen and by $12 \%$ for helium and argon. No such systematic deviation is discernible in Fig. 2.

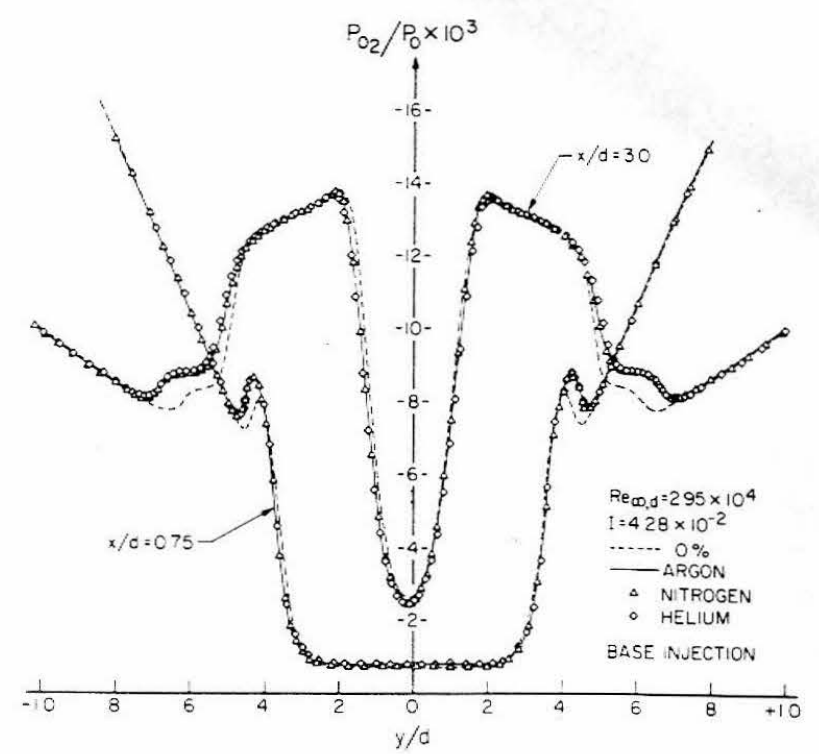

Fig. 5 The correlation of the near-wake flowfield with the mass addition parameter $I$.

Chapman ${ }^{10}$ in that the parameter $I$ is based on the momentum flux of the injected fluid, not the mass flux as proposed by Korst, for example. In addition, the present model assumes that the proper reference conditions are those pertaining to the boundary layer upstream of separation, or more precisely to the reversed flow, rather than the freestream.

\section{Pressure field similitude}

The transverse Pitot-pressure profiles at the neck, shown in Fig. 4 for nitrogen addition at $R e_{\infty, d}=3 \times 10^{4}$, illustrate the major features of the influence of increasing mass addition on the near-wake flowfield. With increasing mass addition, the location of the separation point moves forward on the cylinder $\left(\mathrm{cf} . \mathrm{Herzog}^{7}\right)$, and the separation shock is displaced into the outer, rotational flow with a corresponding increase in strength. In addition, the spreading of the viscous layers results in a pronounced decrease in the strength of the wake, or recompression shock, and the combined effects significantly influence the development of the intermediate- and far-wake flows, downstream of the wake neck.

The correlation of the Pitot-pressure field with the mass addition parameter for argon, nitrogen, and helium addition is shown in Fig. 5 for a single value of $R e_{\infty, d}$ at two selected axial locations; $x / d=0.75$ lies within the recirculating vortex, and $x / d=3.0$ is the location of the wake neck also shown in Fig. 4. The correlation of the entire pressure field, for both values of $R e_{\infty, d}$, has not been included here. This correlation is demonstrated in Ref. 1 where, in addition, the separation and wake shock wave locations are shown as a function of increasing $I$ to conclusively demonstrate the complete pressure field correlation.

\section{Structure of the Mass-Concentration Field}

\section{Near-Wake Flow}

\section{Mass addition from the base}

The results for the near-wake mass-concentration field with mass addition from the base are given in Figs. 6-8. Figure 6 is an isometric plot of the argon mass-concentration field, while Figs. 7 and 8 are isograms of the argon and helium mass-concentration fields, respectively, overlayed on the characteristic features of the corresponding pressure field. In each of these figures the mass-concentration field is dis- 


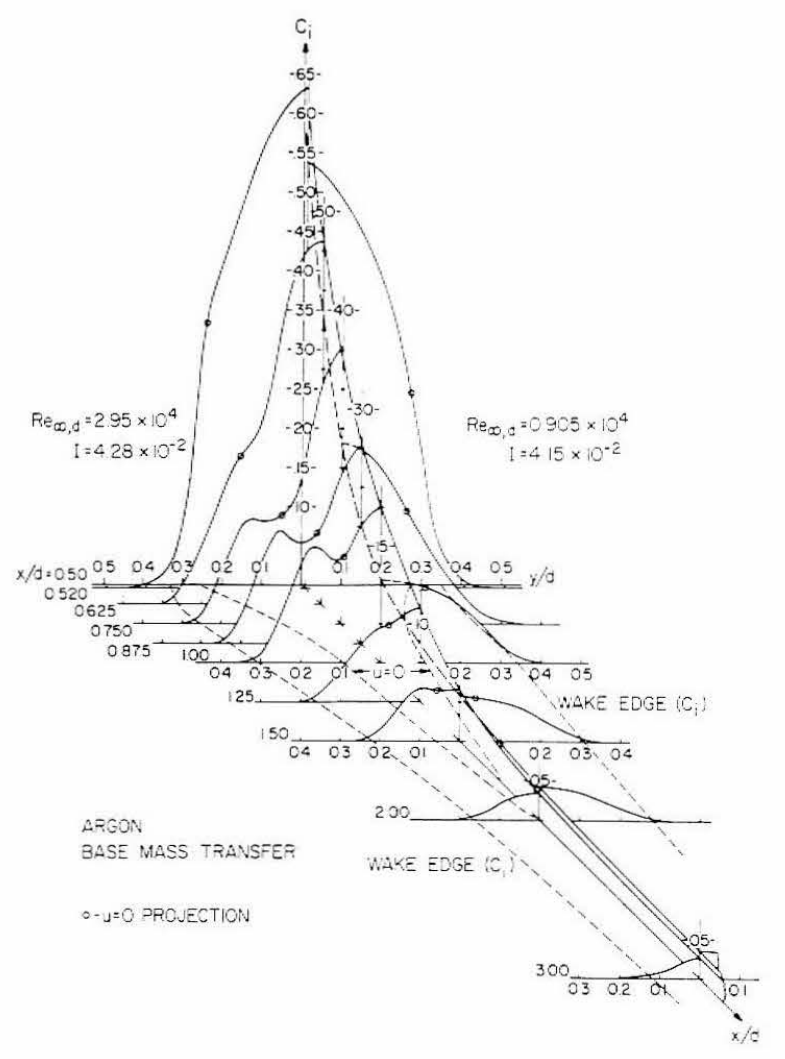

Fig. 6 The near-wake mass-concentration field for argon addition from the base.

played for the same value of $I$ and for each of the two values of Reynolds number.

Figures 6 and 9 demonstrate that the dominant feature of the near-wake mass-concentration field is the axial decay of the mass concentrations from the base toward the rear stagnation point as a result of the counter-current diffusion of the injected species into the reversed flow. A second important aspect of the mass-concentration field is the existence of an outer transport layer, shown in Fig. 6. This outer layer occurs in the vicinity of the shear layers and governs the transport of mass between the recirculating vortex and the outer flow by diffusion across the shear layers, thus establishing the outer boundary condition on the inner recirculating flow.

For $R e_{\infty, d}=3 \times 10^{4}$, the transverse mass-concentration profiles illustrated in Fig. 6 are characterized by an off-axis maximum in the vicinity of the $u=0$ locus as a result of the convection of the high mass-concentration layer near the base into the shear layer by the recirculating flow. This maximum appears as a folding of the jsolines in Fig. 7 and isolates the outer transport laver from the mass-concentration field near the axis. Lateral and axial diffusion result both in the decay of this maximum, as the rear stagnation point is approached, and the subsequent development of the profile toward the asymptotic gaussian form downstream of the rear stagnation point. For $R e_{\infty, d}=0.9 \times 10^{4}$, the mass-concentration field is diffusion dominated within the recirculating zone. At this lower Reynolds number, the local maximum does not exist and the outer transport layer is no longer distinct (Figs. 6, 7). The decreased Schmidt number for helium (Fig. 8) results similarly in the increased role of diffusion, a consequent reduction of the off-axis maximum for $R e_{\infty, d}=3 \times 10^{4}$, and a more rapid transformation to a monotonic diffusion profile.

From this brief description of the mass-concentration field, a close analogy can be seen between the present experiments and the theoretical model proposed by Scott and Eckert ${ }^{19}$ for heat transfer in high Reynolds number separated flow. Scott and Eckert have postulated the existence of two thin layers to describe the transport of heat between the body and the outer flow. The first layer in their model, a boundary layer at the base, governs the transport of heat or mass at the body surface. In the following discussion, the axial profile given in Fig. 9 will be shown to correspond to a generalization of the concept of a base boundary layer for the low Reynolds numbers encountered in the present experiments. The second layer in the model proposed by Scott and Eckert corresponds closely with the outer transport layer observed in the experiments, and governs the transport of heat or mass between the recirculating vortex and the outer flow. Again, this outer layer may not be thin, as a consequence of the low Reynolds numbers encountered in the experiments.

To obtain a qualitative description of the axial distribution of mass concentration in the region between the two stagnation points, an approximate solution of the species conservation equation on the axis has been obtained (Ref. 1) by retaining both the axial and the transverse diffusion terms in the equation, together with the assumption $\rho D=$ const. Then, if the mass-concentration field near the axis is assumed to be self similar, i.e., $C_{i}=C_{£}(\tilde{X}) F[\tilde{Y} / \sigma(\tilde{X})]$, the axial species conservation equation becomes

$$
d^{2} C_{屯} / d \tilde{X}^{2}+\tilde{U}(\tilde{X}) d C_{屯} / d \tilde{X}+\Delta(\tilde{X}) C_{€}=0
$$

where $\tilde{U}(\tilde{X})=\left[u(\tilde{X}) / u_{\max }\right]\left[\left|u_{\max }\right|\left(x_{r}-x_{b}\right) / D\right]=\operatorname{Re}(\tilde{X}) S c$, and $\Delta(\tilde{X})=F(0)^{\prime \prime} / \sigma(\tilde{X})^{2}$.

For a qualitative understanding of the concentration field, it is sufficient to make the assumption that $\tilde{U}(\tilde{X})$ and $\Delta(\tilde{X})$ are independent of $\tilde{X}$. Then the species equation has the solution

$$
C_{\notin}(\tilde{X})=A e^{(\tilde{U} / 2)(\alpha-1) \tilde{X}}+B e^{-(\tilde{U} / 2)(\alpha+1) \tilde{X}}
$$

where $\alpha=\left(1-4 \Delta / \widetilde{U}^{2}\right)^{1 / 2}$ and where $A, B$ are evaluated

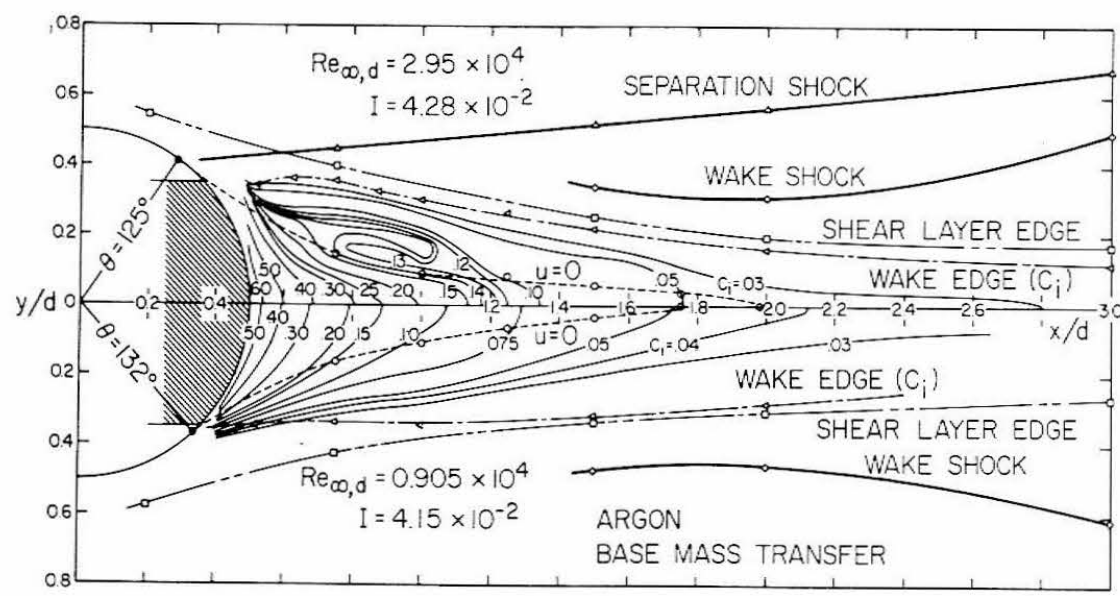

Fig. 7 The near-wake mass-concentration isogram for argon addition from the base. 
Fig. 8 The near-wake mass-concentration isogram for helium addition from the base.

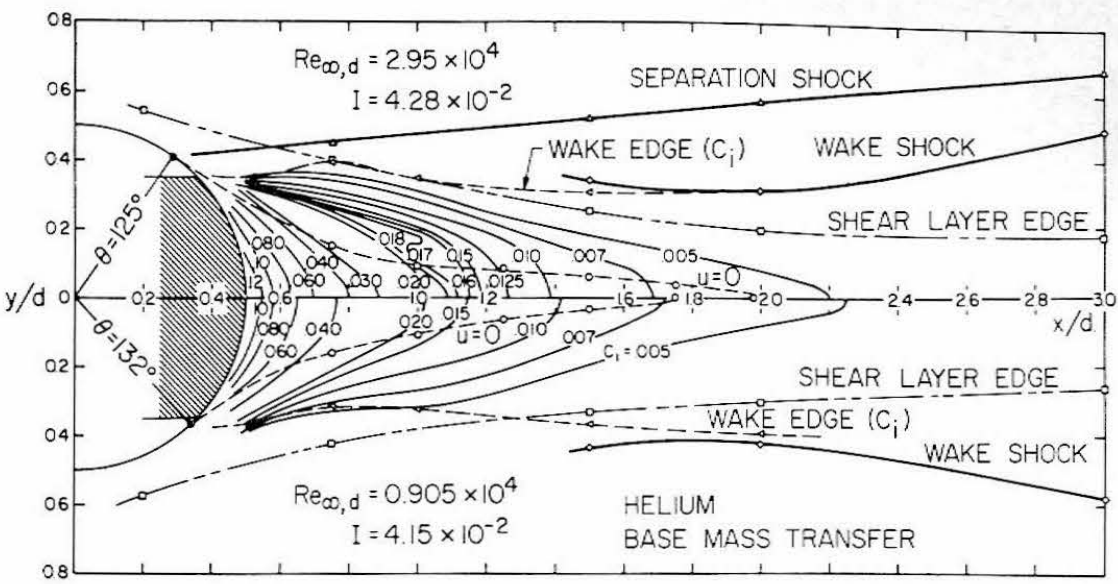

from the boundary conditions at the base and at the rear stagnation point.

It is apparent from these results that the presence of the rear stagnation point imposes as the solution to the species equation the linear superposition of a positive and a negative exponential function. The negative exponential corresponds to the decay from a source at the base stagnation point into a uniform flow of infinite extent with lateral diffusion; whereas, the positive exponential represents the decay toward the base of the mass concentration supplied by a source at the rear stagnation point, whose strength is determined by the outer flow.

As $\tilde{C}=\operatorname{ReS} c \rightarrow \infty$, the boundary-layer character of the solution becomes evident. In this limit $\alpha \approx 1$, and the solution becomes

$$
C_{屯}(\tilde{X})=C_{£}(0) \frac{[e-\tilde{U} \tilde{X}-e-\tilde{U}]}{[1-e-\tilde{U}]}+C_{£}(1) \frac{[1-e-\tilde{U} \tilde{X}]}{[1-e-\tilde{U}]}
$$

Thus, for large Reynolds numbers, the injected species is confined to a thin, exponentially decaying boundary layer with a characteristic thickness $\delta_{b} \sim \bar{U}^{-1}$. This solution corresponds to the thin base boundary layer proposed by Scott and Eckert. ${ }^{19}$

In the present experiments, $\tilde{U}=\operatorname{ReS} c \sim 0(1)$, and the axial diffusion depth is of the order of the distance between the two stagnation points, $\delta_{b} \sim \widetilde{U}^{-1} \approx 1$. Under these circumstances, the near-wake axial distribution given in

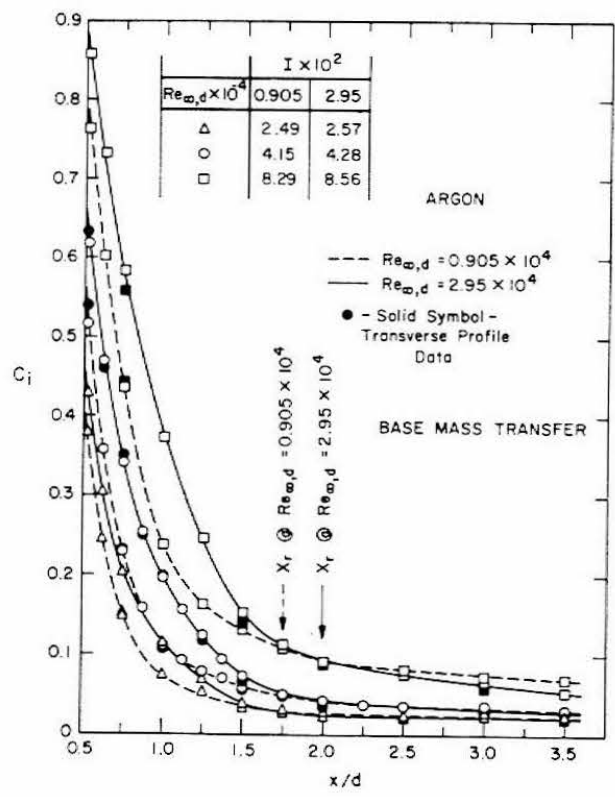

Fig. 9 The axial distribution of mass concentration for argon addition from the base.
Figs. 6 and 9 represents a generalization to low values of $\tilde{U}=\operatorname{ReSc}$ of the concept of a thin base boundary layer proposed by Scott and Eckert. The two length scales in the solution, $\tilde{X} \sim \tilde{U}^{-1}$ and $\tilde{X} \sim(\Delta)^{-1 / 2} \sim \sigma /\left(F(0)^{\prime \prime} \mid\right)^{1 / 2}$ are derived from the influence of counter-current diffusion and of transverse diffusion, respectively.

\section{Forward injection}

For argon addition from the forward stagnation region, the near-wake mass-concentration field is illustrated in the isometric plot in Fig. 10 and the isogram in Fig. 11. In both figures, the same value of the mass transfer parameter $\dot{M}=\dot{m}_{i} / 2 \dot{m}_{B . L}$. is used at each of the two values of Reynolds number.

The species transferred from the forward stagnation region enter the near-wake flowfield through mass-concentration boundary layers whose thickness upstream of separation is comparable to the viscous boundary-layer thickness. The mass-concentration distribution in these boundary layers dominates the boundary conditions for the distribution of mass concentration in the near-wake flowfield, and sets the mass-concentration level at the base. The extensions of the boundary layers into the near-wake flow are the narrow masstransport layers shown in Fig. 10. These layers form the outer transport layers in the two layer model proposed by Scott and Eckert, ${ }^{19}$ and thus provide the outer boundary conditions on the mass-concentration field in the region of reversed flow.

The transverse mass-concentration profiles for argon exhibit a nearly uniform region between the $u=0$ loci (Fig.

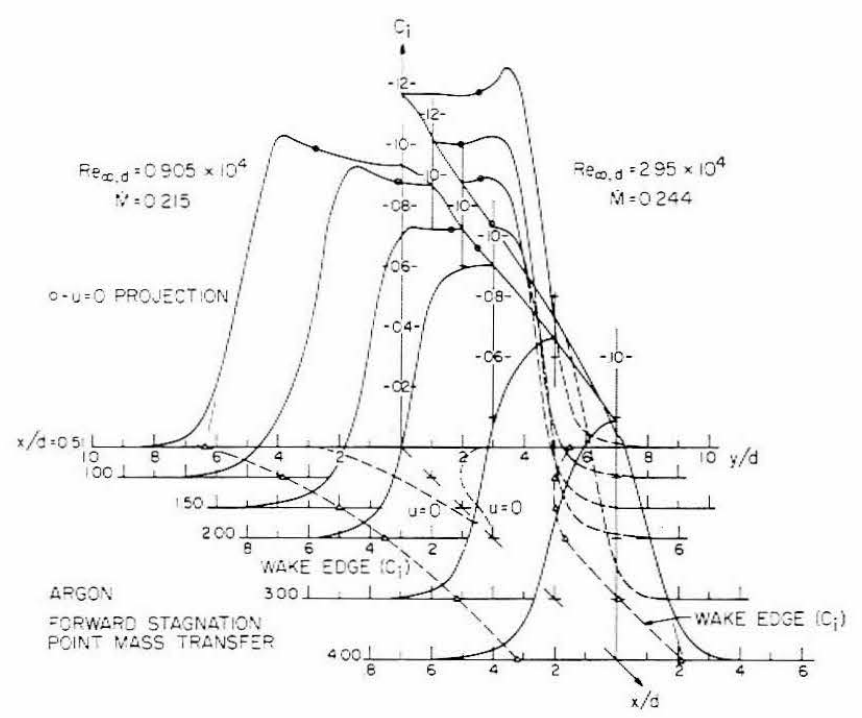

Fig. 10 The near-wake mass-concentration field for argon addition from the forward stagnation region. 


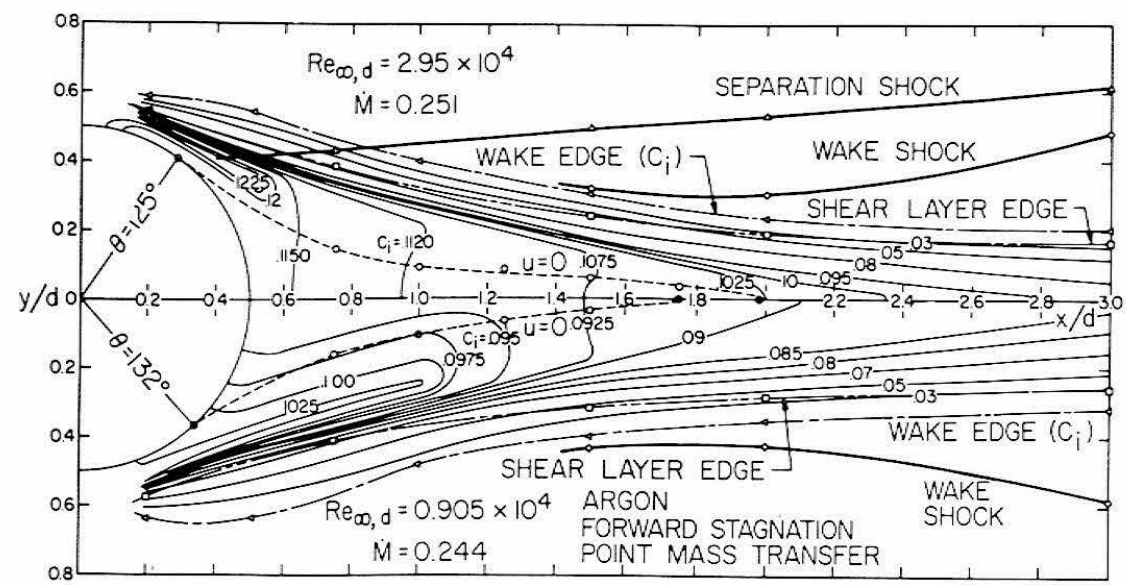

Fig. 11 The near-wake mass-con-centration isogram for argon addition from the forward stagnation region.

11) as a consequence of the effects of transverse diffusion within the recirculating vortex and of the decrease in the mass concentration along the undisturbed dividing streamlines by the diffusional loss of the injected species through the outer transport layers. This latter fact results in a reduction of the mass-concentration level at the rear stagnation point below that at the base and provides the second boundary condition for the axial decay of mass concentration in the near wake. A local maximum occurs off the axis, followed by a rapid decay of the mass concentration in the outer transport layer. The local maximum also occurs in the boundary-layer profiles in the immediate vicinity of separation, but does not persist further upstream.

The near-wake mass-concentration profiles for helium exhibit the dominant role of diffusion. For $R e_{\omega, d}=3 \times 10^{4}$, a local maximum again occurs near the base, but decays rapidly downstream because of the decreased Schmidt number. For $R e_{\infty, d}=0.9 \times 10^{4}$, the profiles exhibit a single inflection point similar to the profiles shown in Fig. 6 for argon addition from the base at the same Reynolds number. The details are given in Ref. (1).

To obtain a qualitative understanding of the mass-concentration field in the present case, the approximate solution given previously for the axial distribution can again be used. For the present case, because the transverse distribution is nearly uniform, $\Delta=0$ and hence $\alpha=1$. Therefore the solution of the axial species conservation equation has the

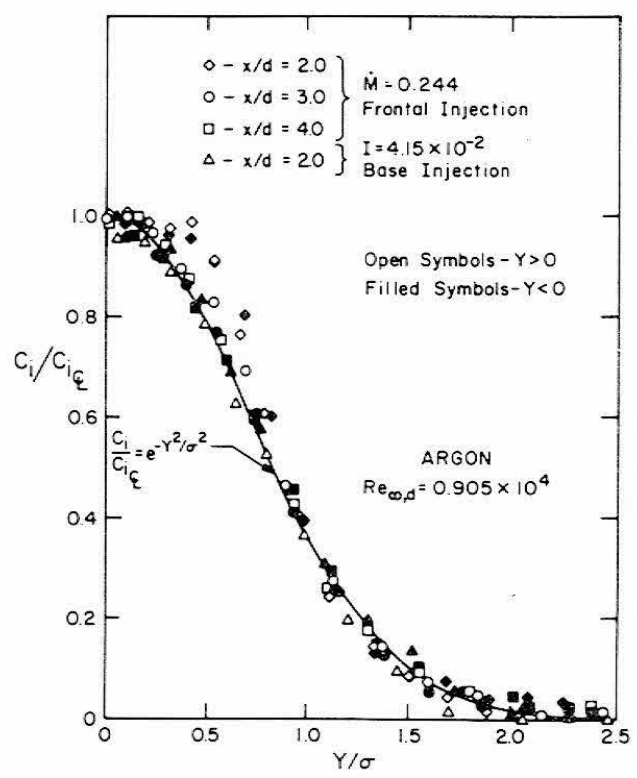

Fig. 12 The normalized distribution of argon mass concentration in the intermediate wake. same form as obtained for $U \rightarrow \infty$ in the previous discussion [Eq. (14)]. However, in the present case, $\widetilde{U}=\operatorname{ReS} c \sim$ $0(1)$ as before, and hence $\delta_{b} \approx 1$, i.e., the axial diffusion depth is of the order of the distance between the two stagnation points. Furthermore, because there is no source in the base region, the difference between the mass-concentrations at the base and at the rear stagnation point results only from the decrease in the mass concentration of the injected species along the dividing streamline by diffusional loss through the outer transport layer. As a result, the two boundary conditions are nearly equal and the axial decay is extremely weak compared to that for base mass addition (cf. Figs. 6, 9, and 10). As $\tilde{U} \rightarrow \infty$, the exponential functions again represent a thin boundary layer at the base. However, because no source exists at the base, the massconcentration field is not expected to exhibit a boundary layer behavior. On the contrary, as $\tilde{U}$ becomes large, convection will dominate over diffusion and it is expected that the mass concentration of the injectant will be uniformly distributed within the recirculating flow, i.e., $C_{\Phi}(1) \rightarrow$ $\mathrm{C}_{\Phi}(0)$.

\section{Intermediate Wake}

When the Oseen linearization used by Kubota ${ }^{20}$ to determine the asymptotic self-similar form for the inner wake is extended to the mass-concentration wake, the asymptotic far-wake solution corresponds to the diffusion from a delta function source at the origin,

$$
C_{i}=\frac{\dot{m}_{i}}{2 \rho_{\infty} u_{\infty} d}\left[\frac{C_{\infty} R e_{\infty}, d s c}{\pi}\right]^{1 / 2} \frac{e^{-S c Y^{2} / 4 X}}{[X]^{1 / 2}}
$$

where the details of the near-wake may be lumped in the description of a virtual origin of the axial coordinate.

The presence of the rear stagnation point, and the finite width of the mass-concentration profiles at the neck, require that there be an intermediate-wake region in which the mass-concentration and velocity fields experience a transition from those distributions imposed at the rear stagnation point by the near-wake flow to their respective asymptotic far-wake distributions. The boundary conditions for the intermediate wake solution are specified by the mass-concentration distribution at the neck. In the incompressible plane, Fig. 12 shows that for argon the measured transverse mass-concentration profiles in the vicinity of the neck rapidly approach a Gaussian form with increasing axial distance. These profiles exhibit a characteristic width $Y=\sigma(X)$, for which $C_{i} / C_{屯}=1 / e$, and their amplitudes $C_{\Phi}$ scale linearly with increasing mass transfer. In addition, the experiments indicate that the lateral scale for the mass-concentration wake depends mechanically on the lateral scale for the viscous wake, independent of the mass transfer rate. 
Using these experimental observations, an approximate solution for the axial distribution of argon mass concentration will be obtained for $R e_{\infty, d}=0.9 \times 10^{4}$. This result is not extended to the data for $R e_{\infty, d}=3 \times 10^{4}$ because of the occurrence of transition in the intermediate-wake flow field at this Reynolds number. The case of helium addition will be mentioned separately.

Neglecting axial diffusion, the species conservation equation on the axis becomes

$$
(u / D) \partial C / \partial x=\partial^{2} C / \partial y^{2}
$$

In addition, conservation of the injected species provides the integral relation

$$
\dot{m}_{i}=2 \int_{0}^{\infty} \rho u C_{i} d y=\text { const }
$$

Using the modified Howarth transformations to the incompressible plane, these equations take the form

$$
\begin{aligned}
& S c U \partial C / \partial X=\partial^{2} C / \partial Y^{2} \\
& \int_{0 N}^{\infty} U C d Y=\int_{0 X}^{\infty} U C d Y
\end{aligned}
$$

where $S c=\nu / D=$ const.

To simplify the calculations, it is convenient to assume that both the mass-concentration and the velocity fields have Gaussian distributions, and possess the same scale, i.e.,

$$
C / C_{\complement}(X)=(1-U) /\left[1-U_{\complement}(X)\right]=e^{-Y^{2} / \sigma^{2}(X)}
$$

To approximate the axial velocity distribution, an analytic form which has the proper limiting behavior for small $X$,

$$
U_{\varepsilon}=X /(a+X)
$$

has been obtained by matching the experimental data at $X=4.0$. Using this relation, if the origin and the boundary conditions are taken at the neck, then defining $\xi=X-X_{N}$, the solution becomes

$$
\begin{array}{r}
\frac{C_{\Phi}(\xi)}{C_{X}}=\left\{1+\frac{8}{S c \sigma_{N}^{2}\left[b+U_{N}\right]^{2}}\left[\xi-\frac{a}{2} \ln \left(\frac{a+X_{n}+\xi}{a+X_{N}}\right)+\right.\right. \\
\left.\left.\left(\frac{3}{2}-[2]^{1 / 2}\right) a \ln \left(\frac{X_{N}+\xi}{X_{N}}\right)\right]\right\}^{-1 / 2}
\end{array}
$$

where $\sigma_{N}, U_{N}$ refer to the length scale and the centerline velocity of the self-similar profile at the neck, and $b=$ $\left(2^{1 / 2}-1\right)$ for a Gaussian profile. For small $\xi$,

$$
C_{\Phi} / C_{N} \rightarrow 1-\beta \xi
$$

For large $\xi$,

$$
\frac{C_{\Phi}}{C_{N}} \rightarrow\left(\beta^{*} \xi\right)^{-1 / 2}, \quad \text { where } \beta^{*}=\frac{8}{S c \sigma_{N}{ }^{2}\left[b+U_{N}\right]^{2}}
$$

Thus, for large $\xi$ the solution approaches the decay law given by the Oseen linearized solution. The virtual origin is then given as $\xi_{0}=1 / \beta^{*} \sim S c \sigma_{N^{2}}$, and is shown by the extrapolation of the solution curve in Fig. 13.

The normalized solution contains a single parameter $\sigma_{N}$, the scale of the transverse profile at the neck. This parameter depends on the distribution in the near-wake flowfield, and has been obtained from experiment by matching the data at an arbitrary value of $\xi$. The results are shown in Fig. 13 for argon addition from both the forward and the base stagnation regions.

For helium addition, the near-field solution for $\xi \rightarrow 0$ is invalid because the axial diffusion term is not negligible near the origin. The far-field solution is valid, however, and has been used in Ref. 1 to further substantiate dependence of the virtual origin on $S c \sigma_{N}{ }^{2}$ for the asymptotic far wake.

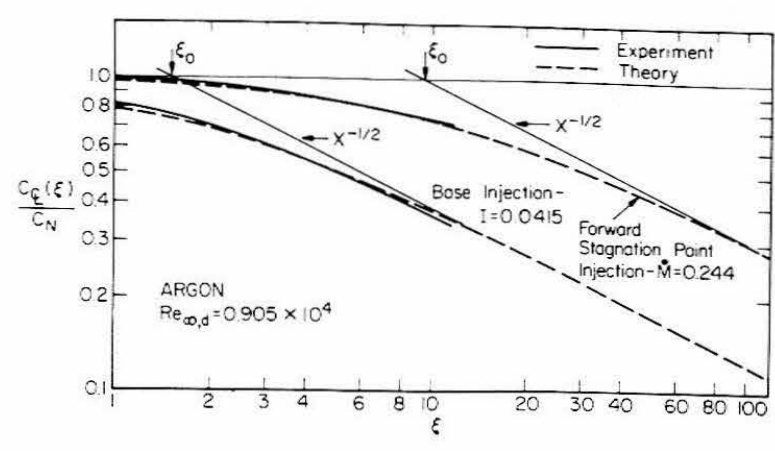

Fig. 13 The axial mass-concentration distribution of argon in the intermediate wake.

\section{Summary}

An experimental investigation of the steady, laminar nearwake flowfield of a two-dimensional, adiabatic, circular cylinder with surface mass transfer has been made at a freestream Mach number of 6.0 , and freestream Reynolds numbers $R e_{\infty, d}=0.9$ and $3.0 \times 10^{4}$.

Mass addition from the forward stagnation region has no measurable influence on the near-wake pressure field for moderate mass transfer rates.

For mass addition from the base, the present experiments conclusively demonstrate that the base pressure, and the entire near-wake flowfield, correlates with the parameter

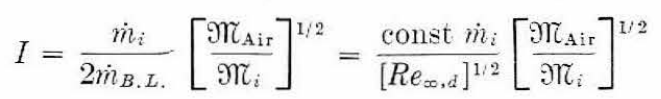

the ratio of the momentum flux of the injected fluid to the momentum flux in the cylinder boundary layer upstream of separation, and not the mass flux as proposed by Korst. Indeed, whether any such regime exists in which the nearwake flowfield correlates with the mass flux of the injectant is not clear. Such a regime does not exist in the present measurements even for vanishingly small changes in base pressure. The mechanism which determines the behavior of the base pressure with mass addition is the establishment of a stagnation point off the base, formed by the balance of momentum between the injected fluid and the reversed flow, and the consequent impression of the injected gas on the free shear layer near separation.

In view of the present experiments, it is clear that an investigation of the effects of molecular weight is crucial to distinguish the roles of mass flux, momentum flux, and volume flux. For this reason, some of the results reported by Carriére ${ }^{12}$ and by Ginoux, ${ }^{13}$ i.e., appear to be inconclusive in this regard.

For mass addition from the base, the base mass concentration is large, and the dominant feature of the massconcentration field is the rapid axial decay away from the base as a consequence of the counter-current diffusion of the injected species into the reversed flow.

For mass addition from the forward stagnation region, because no source exists at the base, the mass-concentration field is nearly uniform in the region of reversed flow, and possesses a local maximum in the vicinity of the $\psi=0$ streamline.

In the intermediate-wake region, downstream of the neck, the mass-concentration field has been examined with the aid of an approximate theoretical model which assumes that the mass-concentration distribution is Gaussian in the incompressible plane. For argon addition, the axial diffusion terms are negligible and the species conservation equation on the axis gives a representation for the axial decay. By comparison with the experimental data, the solution yields the axial distribution, and the location of the virtual origin for the asymptotic far-wake solution, as a function of the distribution of mass concentration at the wake neck. 


\section{References}

${ }^{1}$ Collins, D. J., "The Near Wake of a Two-Dimensional Hypersonic Blunt Body with Mass Addition," Ph.D. thesis, Dec. 1968, California Institute of Technology, Pasadena, Calif.

${ }^{2}$ Reeves, B. L. and Lees, L., "Theory of Laminar Near Wake of Blunt Bodies in Hypersonic Flow," AIAA Journal, Vol. 3, No. 11, Nov. 1965, p. 2061.

${ }^{3}$ Klineberg, J. M., "Theory of Laminar Viscous-Inviscid Interactions in Supersonic Flow," Ph.D. thesis, June 1968, California Institute of Technology, Pasadena, Calif.

${ }^{4}$ McCarthy, J. F. and Kubota, T., "A Study of Wakes behind a Circular Cylinder at $M=5.7, "$ AIAA Journal, Vol. 2, No. 4, April 1964, p. 629.

5 Mohlenhoff, W., "Experimental Study of Helium Diffusion in the Wake of a Circular Cylinder at $M=5.8$," GALCIT Hypersonic Research Project Memo. 54, May 1960, California Institute of Technology, Pasadena, Calif.

${ }^{6}$ Kingsland, L., "Experimental Study of Helium and Argon Diffusion in the Wake of a Circular Cylinder at $M=5.8$," GALCIT Hypersonic Research Project Memo. 60, June 1961, California Institute of Technology, Pasadena, Calif.

' Herzog, R. T., "Nitrogen Injection into the Base Region of a Hypersonic Body," GALCIT Hypersonic Research Project Memo. 71, Aug. 1964, California Institute of Technology, Pasadena, Calif.

${ }^{8}$ Korst, H. H., Page, R. H., and Childs, M. E., "A Theory for Base Pressures in Transonic and Supersonic Flow," M.E. TN-392-2, March 1955, Mechanical Engineering Department, University of Illinois, Urbana, Ill.; also, Journal of Applied Mechanics, Vol. 23, Dec. 1956, p. 593.

${ }^{9}$ Chapman, D. R., Kuehn, D. M., and Larson, H. K., "Investigation of Separated Flows in Supersonic and Subsonic
Streams with Emphasis on the Effects of Transition," TN-1356, 1958, NACA.

${ }^{10}$ Chapman, D. R., "Theoretical Analysis of Heat Transfer in Separated Flows," TN-3792, 1956, NACA.

${ }_{11}$ Davis, L. R., "Experimental and Theoretical Determination of Flow Properties in a Reacting Near Wake," AIAA Journal, Vol. 6, No. 5, May 1968, p. 843.

${ }^{12}$ Carriére, P., "Recherches Récentes Effectuées A L’O.N.E.R.A. Sur les Problémes de Recollement," T.P. 27a, 1965, O.N.E.R.A., France.

${ }^{13}$ Ginoux, J. J., "Effects of Gas Injection in Separated Supersonic Flows," TCEA TN-7, Feb. 1962, Von Kàrmàn Institute, Rhode-Saint-Genese, Belgium.

${ }^{14}$ Lewis, J. E. and Behrens, W., "Fluctuation Measurements in the Near Wake of a Wedge with and without Base Injection," AIAA Journal, Vol. 7, No. 4, April 1969, p. 664.

${ }^{15}$ Reis, V. H. and Fenn, J. B., "Separation of Gas Mixtures in Supersonic Jets," Journal of Chemistry and Physics, Vol. 39, No. 12, Dec. 1963 , p. 3240.

${ }^{16}$ Fuller, L. and Reid, J., "Experiments on Two-Dimensional Base Flow at $M=2.4, "$ R\&M 3064, 1958, Aeronautical Research Council, London, England.

${ }^{17}$ Chapman, D. R., "Laminar Mixing of a Compressible Fluid," TN-1800, Feb. 1949, NACA; also Rept. 958, 1950, NACA. 18 Shreeve, R. P., "Supersonic Flow from a Porous Metal Plate," AIAA Journal, Vol. 6, No. 4, April 1968, p. 752.

${ }^{19} \mathrm{Scott}, \mathrm{C}$. J. and Eckert, E. R. G., "Heat and Mass Exchange in the Supersonic Base Region," AGARD Conference Proceedings 4, Separated Flows. Pt. I, May 1966, p. 429.

${ }^{20}$ Kubota, T., "Laminar Wake with Streamwise Pressure Gradient," GALCIT Hypersonic Research Project, Internal Memo. 9, May 1962, California Institute of Technology, Pasadena, Calif. 